\title{
The prospective evaluation of malnutrition in hospitalized children in a pediatric urology unit
}

\author{
Sebahat $\mathrm{CAM}^{1}$ (D), Tarik Emre SENER ${ }^{2}$ (1) \\ Divison of Pediatric Gastroenterology, Department of Pediatrics, School of Medicine, Istanbul Medeniyet University, Istanbul, Turkey \\ 2 Department of Urology, School of Medicine, Ministry of Health-Marmara University Training and Research Hospital, Pendik, Istanbul, Turkey \\ Corresponding Author: Sebahat CAM E-mail: imamoglus@yahoo.com
}

Submitted: 22.07.2019 Accepted: 13.09.2019

\begin{abstract}
Objective: Malnutrition has been reported to be a rather common health problem in hospitalized children and it is recommended to be evaluated in these conditions. The objective of this prospective trial was to evaluate the nutritional status of hospitalized pediatric urology patients and find the prevalence of malnutrition in this specific subgroup of patients.

Patients and Methods: All subsequent children between 1 and 18 years old who were hospitalized between January 2018 and January 2019 in the pediatric urology unit of a referral university hospital were included into the study. STRONGkids questionnaire was used to assess the nutritional status of the children. Malnutrition was evaluated according to anthropometric measurements based on the computer programs developed by the World Health Organization (WHO).

Results: A total of 76 patients were included in the study. The mean age of the patients was $8.84 \pm 4.23$; mean height was $121.03 \pm 22.49$ $\mathrm{cm}$; mean weight was $27.8 \pm 13.41 \mathrm{~kg}$. Malnutrition risk, according to STRONGkids screening tool was high in 1 patient, medium in 8 patients and low in 67 patients. According to anthropometric measurements, 6 patients (7.9\%) and 25 patients (32.9\%) had acute and chronic malnutrition, respectively.

Conclusion: The current prospective trial suggested that malnutrition is a significant issue also for hospitalized pediatric urology patients.

Keywords: Malnutrition, Pediatric Urology, STRONGkids, Hospital
\end{abstract}

\section{INTRODUCTION}

Malnutrition is a serious condition and it accounts almost half of the deaths under 5 years of age [1]. Therefore, malnutrition requires an appropriate assessment particularly in pediatric population. By definition, malnutrition denotes development of clinical problem in tissues, body shape, and functions related with inadequate intake of energy supplying foods (carbohydrates and fats), protein, and micronutrients (vitamins and minerals) $[1,2]$. It also adversely affects immune system and the patient may become prone to infections [2]. Consequently, infections enhance the degree of malnutrition. Particularly, malnutrition has been defined as a significant issue for hospitalized children according to a variety of different studies [3-5]. The prevalence of malnutrition for hospitalized children was observed in $6.1 \%$ to $40.9 \%$ in a multi-centric trial including different countries in 2008 [5]. In this study, malnutrition had an incidence between 6 to $14 \%$ in hospitalized children even in well-known developed countries. Two university hospitals from Turkey were included in this report. Highest malnutrition rate (ranked $1^{\text {st }}$ ) was reported to be $31.8 \%$ for a hospital in Izmir [6]. Second observation presented a rate of $52.4 \%$ as acute malnutrition among 585 children hospitalized at a university hospital in Istanbul [7]. A recent study reported that the prevalence of malnutrition was $13.4 \%$ in a pediatric surgery unit in Istanbul [8]. All these figures suggest that hospitalized children should be evaluated for malnutrition. Different pediatric units may have different malnutrition rates that the researchers should bear in mind. Establishment of these differences in malnutrition rates in various subgroups of pediatric patients carries clinical significance. It should be considered that an appropriate nutrition treatment is required for malnutrition particularly in hospitalized children [9]. Furthermore, hospitals should establish nutritional care teams to assess malnutrition in hospitalized children, and these teams should also provide a particular management protocol on malnourished children.

There is no particular data about the prevalence of malnutrition specific to pediatric urology in-patient clinics. These units mostly deal with vesicoureteral reflux (VUR), urinary tract infection (UTI) and urinary system stone disease in hospitalized patients. Penile or inguinal surgeries constitute the majority of remaining patient population. Therefore, determination of

How to cite this article: Cam S, Sener TE. The prospective evaluation of malnutrition in hospitalized children in a pediatric urology unit. Marmara Med J 2019; 32: 118-123. doi: 10.5472/marumj.637066 
malnutrition prevalence in this specific subgroup of pediatric patients would be valuable for clinical practice.

There are different validated forms to evaluate malnutrition for pediatric hospitalized patients. Among them Screening Tool for Risk of Impaired Nutritional Status and Growth (STRONGkids) is the most widely used one [10]. This questionnaire was developed to screen malnutrition for hospitalized pediatric patients between 1 month to 18 years of age.

The objective of this prospective trial was to evaluate the prevalence of malnutrition in a pediatric urology unit of a referral university hospital.

\section{PATIENTS and METHODS}

This prospective trial was approved by the local ethical committee (Approval number 09.2018.402). All pediatric patients between 1 month to 18 years of age were included. The malnutrition assessment was provided by a single physician at the day of hospital admission. Demographic properties, medical history, primary diagnosis, and routine laboratory tests of all cases were recorded. The patients with diabetes mellitus, previous gastrointestinal surgery, celiac disease, neurological deficit and any pediatric malignancy were classified separately.

Weight and height were recorded as anthropometric measurements to evaluate malnutrition by the same physician. The World Health Organization (WHO) Anthro and AnthroPlus Programs were used for the assessment of these results $[11,12]$. For acute malnutrition (severe or moderate), "Weight-forHeight (WFH) SD (standard deviation) score" or "Body Mass Index (BMI) SD score" less than - 2 was utilized [5]. Chronic malnutrition was defined as "Height-for-Age (HFA) SD score" less than - 2 [5]. Moreover, to calculate SD different " $\mathrm{z}$-scores" (WFH, BMI, HFA) were calculated using the WHO programs.

Validated STRONGkids questionnaire was applied to evaluate malnutrition risk. Similarly, all assessments were performed by the single physician without knowing the clinical diagnosis.

\section{Statistical Analyses}

Statistical analyses were performed using SPSS Software (IBM SPSS Statistics version 20.0, SPSS Inc. Chicago, IL). Categorical variables were summarized as percentages and were compared using either Fisher's exact or Chi-Square tests. A p $<0.05$ was considered statistically significant.

\section{RESULTS}

A total of 76 patients (36 girls, 40 boys) were included. Five patients with known concomitant diseases associated with malnutrition were excluded from the study. These diseases were type 1 diabetes mellitus in one patient, celiac disease in one patient, cerebral palsy in two patients, and recent intestinal surgey in one patient. The mean age for girls was $9.23 \pm 4.25$ years, and was $8.49 \pm 4.24$ years for boys. Hospitalization reasons were urinary system stone disease in $34.2 \%$ (n:26) of the cases. The remaining indications were anti-reflux surgery for vesicoureteral reflux (VUR) in $22.4 \%$ (n:17), urinary tract infection (UTI) due to neurogenic bladder in $14.5 \%$ (n:11), UTI associated with VUR in $18.4 \%$ (n:14), penile-inguinal or scrotal surgeries in $10.5 \%$ (n:8) (Table I). The assessments were performed at the day of hospital admission. The mean duration of hospitalization was $4 \pm 1.1$ days.

Table I. Demographic data and hospitalization indications

\begin{tabular}{|l|l|}
\hline Age (Mean+ SD) year & $\begin{array}{l}\text { Hospitalized pediatric } \\
\text { urology patients (n=76) }\end{array}$ \\
\hline Sex (Male/Female, $\mathbf{n}, \%)$ & $8.84 \pm 4.23$ \\
\hline Urinary stone surgery (n,\%) & $40 / 36,52.6 \% / 47.4 \%$ \\
\hline Anti-reflux surgery (n,\%) & $26(34.2 \%)$ \\
\hline UTI with neurogenic bladder (n,\%) & $17(22.4 \%)$ \\
\hline UTI with VUR (n,\%) & $11(14.5 \%)$ \\
\hline Inguinoscrotal and penile surgery $(\mathbf{n}, \%)$ & $14(18.4 \%)$ \\
\hline
\end{tabular}

(VUR: Vesicoureteral reflux; UTI: Urinary tract infection)

Anthropometric measurements revealed acute malnutrition in 6 cases $(6 / 76 ; 7.9 \%)$, and chronic malnutrition in 25 patient (25/76; 32.9\%) (Tables II and III). The overall clinical diagnosis of the children regarding acute and chronic malnutrition was urinary stone disease in $2.6 \%$ and $7.9 \%$ of the cases, respectively. Hospitalization indication was VUR with acute and chronic malnutrition in $2 \%$ and $10.5 \%$ of the patients, respectively (Table III). Remaining hospitalization indications and malnutrition rates were shown in table III. The number of patients with penile and inguinoscrotal surgeries was low. On the other hand, the rate of acute and chronic malnutrition varied with respect to the hospitalization indications (Table IV). The rate of acute malnutrition was $11.8 \%$ while chronic malnutrition was $47 \%$ in children with VUR surgery. Similarly, $9 \%$ and $45.5 \%$ of children with antibiotic treatment for UTI due to the neurogenic bladder dysfunction had acute malnutrition and chronic malnutrition, respectively. The relationship between the hospitalization indication and malnutrition was shown in Table IV. However, no statistically significant difference in acute malnutrition regarding the clinical diagnosis was observed $(p=0.990)$. Similarly, the incidence of chronic malnutrition was not significant with respect to the hospitalization indication $(\mathrm{p}=0.432)$.

Table II. Anthropometric measurements

\begin{tabular}{|l|l|}
\hline Measurement & $\begin{array}{l}\text { Hospitalized pediatric urology } \\
\text { patients } \\
(\mathbf{n}=76) \\
\text { Mean }( \pm \mathrm{SD})\end{array}$ \\
\hline Weight $(\mathbf{k g})$ & $27.8 \pm 13.41$ \\
\hline Height $(\mathbf{c m})$ & $121.03 \pm 22.49$ \\
\hline Body mass index $(\mathbf{k g} / \mathrm{m} 2)$ & $17.89 \pm 3.49$ \\
\hline Weight-for-Age z score & $-0.67 \pm 1.11$ \\
\hline Height-for-Age z score & $-1.38 \pm 1.22$ \\
\hline Body Mass Index for Age z score & $0.15 \pm 1.27$ \\
\hline
\end{tabular}


Table III. The distribution of malnutrition prevalence based on anthropometric measurements according to hospitalization indications

\begin{tabular}{|c|c|c|c|c|}
\hline \multirow{2}{*}{ Hospitalization indications $(\mathrm{n}=76)$} & \multicolumn{2}{|c|}{ Acute Malnutrition } & \multicolumn{2}{|c|}{ Chronic Malnutrition } \\
\hline & $+(\mathrm{n}, \%)$ & $-(\mathrm{n}, \%)$ & $+(\mathrm{n}, \%)$ & $-(\mathbf{n}, \%)$ \\
\hline Urinary stone surgery $(\mathrm{n}, \%)$ & $2(2.6 \%)$ & $24(31.6 \%)$ & $6(7.9 \%)$ & $20(26.3 \%)$ \\
\hline Anti-reflux surgery $(\mathrm{n}, \%)$ & $2(2.6 \%)$ & $15(19.7 \%)$ & $8(10.5 \%)$ & $9(11.8 \%)$ \\
\hline UTI with neurogenic bladder (n,\%) & $1(1.3 \%)$ & $10(13.2 \%)$ & $5(6.6 \%)$ & $6(7.9 \%)$ \\
\hline UTI with VUR (n,\%) & $1(1.3 \%)$ & $13(17.1 \%)$ & $4(5.3 \%)$ & $10(13.2 \%)$ \\
\hline Inguinoscrotal and penile surgery (n,\%) & 0 & $8(10.5 \%)$ & $2(2.6 \%)$ & $6(7.9 \%)$ \\
\hline Total & $6 / 76(7.9 \%)$ & $70 / 76(92.1 \%)$ & $25 / 76(32.9 \%)$ & $51 / 76(67.1 \%)$ \\
\hline
\end{tabular}

(VUR: Vesicoureteral reflux; UTI: Urinary tract infection)

Tablo IV. The respective prevalence of malnutrition according to hospitalization indications

\begin{tabular}{|c|c|c|c|c|}
\hline \multirow{2}{*}{ Hospitalization indications $(\mathrm{n}=76)$} & \multicolumn{2}{|c|}{ Acute Malnutrition } & \multicolumn{2}{|c|}{ Chronic Malnutrition } \\
\hline & $+(\mathrm{n}, \%)$ & $-(\mathrm{n}, \%)$ & $+(\mathrm{n}, \%)$ & $-(\mathrm{n}, \%)$ \\
\hline Urinary stone surgery (n:26) & $2 / 26(7.7 \%)$ & $24 / 26(92.3 \%)$ & $6 / 26(23.1 \%)$ & $20 / 26(76.9 \%)$ \\
\hline Anti-reflux surgery (n:17) & $2 / 17(11.8 \%)$ & $15 / 17(88.2 \%)$ & $8 / 17(47 \%)$ & $9 / 17(53 \%)$ \\
\hline UTI with neurogenic bladder (n:11) & $1 / 11(9 \%)$ & $10 / 11(91 \%)$ & $5 / 11(45.5 \%)$ & $6 / 11(54.5 \%)$ \\
\hline UTI with VUR (n:14) & $1 / 14(7.1 \%)$ & $13 / 14(92.9 \%)$ & $4 / 14(28.6 \%)$ & 10/14 (71.4\%) \\
\hline Inguinoscrotal and penile surgery $(\mathrm{n}: 8)$ & $0 / 8(0 \%)$ & $8 / 8(100 \%)$ & $2 / 8(25 \%)$ & $6 / 8(75 \%)$ \\
\hline Total & $6 / 76$ & $70 / 76$ & $25 / 76$ & $51 / 76$ \\
\hline
\end{tabular}

(VUR: Vesicoureteral reflux; UTI: Urinary tract infection)

The overall mean STRONGkids score was $0.22 \pm 0.72$ in boys and $0.16 \pm 0.69$ in girls. The mean STRONGkids scores were $0.23 \pm 0.6$ for urinary stone disease surgery, $0.35 \pm 0.86$ for VUR surgery, $0.36 \pm 1.22$ for UTI due to the neurogenic bladder, $0.07 \pm 0.26$ for UTI associated with VUR, and 0 for penile-inguinoscrotal surgeries (Table V). The risk assessment for malnutrition based on STRONGkids score revealed low risk in 67 patients, medium risk in 8 patients, and high risk in only 1 patient.
The incidence of malnutrition according to anthropometric measurements of STRONGkids questionnaire was shown in table VI. Six patients with acute malnutrition belonged to the low risk group based on STRONGkids score. Only 2 cases out of 25 chronic malnutrition patients were in the medium risk group according to the STRONGkids assessment. There was no correlation between the STRONGkids risk groups and anthropometric measurements.

Table V. STRONGkids risk assessments

\begin{tabular}{|l|l|l|l|l|l|}
\hline \multirow{2}{*}{ Hospitalization indications $(\mathbf{n}=\mathbf{7 6})$} & \multicolumn{2}{l}{ Risk Groups } & \multicolumn{2}{l|}{ Acute Malnutrition } & Chronic Malnutrition \\
\cline { 2 - 6 } & Low & $\begin{array}{l}\text { Moderate } \\
(\mathbf{0})\end{array}$ & $\begin{array}{l}\text { High } \\
\mathbf{( 1 - 3 )}\end{array}$ & BMI z score (Mean \pm SD) & $\begin{array}{l}\text { HFA z score } \\
(\mathbf{M e a n} \pm \text { SD) }\end{array}$ \\
\hline Urinary stone surgery & $22(28.9 \%)$ & $4(5,3 \%)$ & 0 & $0.14 \pm 1.16$ & $-1.08 \pm 1.24$ \\
\hline Anti-reflux surgery & $14(18.4 \%)$ & $3(3,9 \%)$ & 0 & $-0.2 \pm 1.46$ & $-1.76 \pm 1$ \\
\hline UTI with neurogenic bladder & $10(13.2 \%)$ & 0 & $1(\% 1,3)$ & $0.15 \pm 1.01$ & $-2.01 \pm 1.12$ \\
\hline UTI with VUR & $13(17.1 \%)$ & $1(1.3 \%)$ & 0 & $0.32 \pm 1.44$ & $-1.3 \pm 1.17$ \\
\hline Inguinoscrotal and penile surgery & $8(10.5 \%)$ & 0 & 0 & $0.62 \pm 1.32$ & $-0.79 \pm 1.51$ \\
\hline
\end{tabular}

(VUR: Vesicoureteral reflux; UTI: Urinary tract infection; BMI: Body Mass Index; SD: Standard Deviation; HFA: Height for age )

Table VI. STRONGkids risk groups according to malnutrition based on anthropometric measurements

\begin{tabular}{|l|l|l|l|l|}
\hline & & \multicolumn{3}{|c|}{ STRONGkids Risk Groups } \\
\hline \multirow{2}{*}{ Acute Malnutrition } & + & Low $(\mathbf{0})$ & Moderate (1-3) & High (4-5) \\
& - & $6(7.9 \%)$ & 0 & 0 \\
\cline { 2 - 6 } & - & $61(80.3 \%)$ & $8(10.5 \%)$ & $1(1.3 \%)$ \\
\hline \multirow{2}{*}{ Chronic Malnutrition } & + & $23(30.3 \%)$ & $2(2.6 \%)$ & 0 \\
\cline { 2 - 6 } & - & $44(57.9 \%)$ & $6(7.9 \%)$ & $1(1.3 \%)$ \\
\hline
\end{tabular}




\section{DISCUSSION}

Malnutrition is a major public health problem with significant consequences [1]. It constitutes a remarkable risk factor for morbidity and mortality especially in children [13]. Malnutrition is also associated with several adverse events including alteration in host immune system, increased infection rate, defective tissue healing process, change in gastrointestinal tract functions, prolonged intubation and longer hospital stays [14]. Moreover, malnutrition causes muscle loss and even depression [15]. All these facts prove that malnutrition is a significant issue for health budget for governments. Besides, presence of malnutrition even in adult patients increases overall mortality rates in one year period [16]. Therefore, a relatively higher incidence of malnutrition in hospitalized children should be regarded as a frightening issue in clinical practice. A wide range of malnutrition incidence from $5 \%$ to almost $50 \%$ has been reported depending on the level of development in various countries [5]. For instance, a rate of $24.1 \%$ for malnutrition was detected in 500 hospitalized children [17]. In this study, some patient groups were defined as high risk for malnutrition. Malnutrition was seen up to $40 \%$ in mentally retarded patients. The occurrence of malnutrition was $34.5 \%$ in children with any infection and $33.3 \%$ in cases with cystic fibrosis [17]. Consequently, this study alone suggests that malnutrition prevalence varies among both different patient units and different diseases. There has been no specific report on malnutrition for pediatric urology units in the literature. Therefore, this prospective study would provide new data about malnutrition rates in this specific subgroup of pediatric patients.

All these observations clearly suggest that the physical examination of hospitalized pediatric patients should include malnutrition assessment. At least a rough nutritional evaluation should not be ignored [18]. On the other hand, a simple and easy method is required to evaluate malnutrition for busy centers. Moreover, this nutritional assessment may be performed by a specially trained nurse. For this purpose, several screening tools have been introduced [17, 19-24]. "Nutrition Risk Score (NRS)", "Pediatric Nutritional Risk Score (PNRS)", "Screening Tool for the Assessment of Malnutrition in Pediatrics (STAMP)", "Subjective Global Nutritional Assessment (SGNA)", "Pediatric Yorkhill Malnutrition Score (PYMS)", "Screening Tool for Risk of Impaired Nutritional Status and Growth (STRONGkids)" were several questionnaires developed for hospitalized children to evaluate nutritional status [24]. Among them STRONGkids is the simple and easy-to-use one requiring no anthropometric measurements. Therefore, STRONGkids is widely utilized and validated into several languages [18, 23 - 25]. In this study we then preferred STRONGkids because of these advantages. This modality was also used as a national nutritional screening modality. The Dutch national survey including 44 hospitals and 424 patients used STRONGkids [25]. In this screening study, anthropometric measurements revealed $19 \%$ of malnutrition, while STRONGkids scores proposed $62 \%$ of moderate to severe malnutrition. They also suggested a higher risk with longer hospital stays. In this study, they also defined high, moderate and low risk groups based on STRONGkids scores as 4-5, 1-3, and
0 , respectively. The authors suggested a dietitian consultation for malnutrition, and nutritional management program in high risk group. For moderate risk group, a close follow-up of twice a week with weight measurement and dietary assessment plus weekly nutritional evaluation were advised. Even weekly follow-up was advised for low risk patients. In this study, $1.3 \%$ of the patients had high and $10.4 \%$ of the cases had moderate risk according to the STRONGkids scores. However, since relatively low number of patients was present in subgroups no specific risk assessment was available regarding the hospitalization indications. Similarly, no correlation between the risk groups and definitive malnutrition rates based on anthropometric measurements has been defined due to again relatively few patients in each subgroup.

In a recent trial including approximately 500 patients from the pediatric surgery unit of a university hospital, $35.7 \%$ of the cases were moderate to severe risk group based on STRONGkids scores [8]. The prevalence of malnutrition was reported to be $13.4 \%$ based on anthropometric measurements. They observed a higher malnutrition rate in children less than 60 months as $16.6 \%$ vs $10 \%$. Overall $76 \%$ of the patients underwent surgery. Our study demonstrated $7.9 \%$ of acute malnutrition, and $32.9 \%$ of chronic malnutrition in the pediatric urology unit. This suggested that different patient groups would have different malnutrition rates. On the other hand, the mean age of the patients in pediatric surgery clinic was about 5.8 years, while in our study it was 8.8 years. Also $67 \%$ of the patients underwent outpatient surgery in the pediatric surgery study. In the current study, majority of the patients were hospitalized for at least 24 hours. About half of the surgeries were for inguinoscrotal or penile diseases in the pediatric surgery unit. This rate was only $10.5 \%$ in our study. Therefore, different characteristics of the patients in these two studies yielded different malnutrition rates. Current trial also proposed that patients with VUR and UTI associated with neurogenic bladder had higher malnutrition rates. Almost half the patients belonged to these subgroups had chronic malnutrition.

A new multi-centric trial from Turkey including 37 hospitals and 1513 patients investigated a malnutrition screening program [26]. They used STRONGkids and "Pediatric Yorkhill Malnutrition Score (PYMS)" questionnaires for screening. Anthropometric measurements were also recorded. At hospital admission, 9.5\% and $11.2 \%$ of malnutrition was detected based on BMI SD scores less than -2 and WFH SD less than -2, respectively. STRONGkids revealed moderate risk in 747 patients and high risk in 54 cases. Presence of a chronic disorder increased the risk. A chronic disease was present in about $90 \%$ of the 54 children with high risk. However, PYMS proposed a larger high risk group as 365 patients. They concluded that the correlation of STRONGkids and PYMS results with anthropometric measurements greatly varied depending on age groups. They calculated different specificity and sensitivity values for malnutrition screening tools for different subgroups. Therefore, they suggest that every pediatric patient should have a comprehensive evaluation for malnutrition including anthropometric measurements and a screening tool. In current trial there was no correlation between 
anthropometric measurements and STRONGkids risk groups due to low number of subgroup patients. Nevertheless, we also detected chronic malnutrition in 1/3 of the cases. Moreover, some disorders such as VUR constitute a risk factor for malnutrition based on anthropometric measurements. Therefore, we also strongly advised a malnutrition evaluation that must include anthropometric measurements besides a screening tool. On the other hand, it was shown that there is a probability of significant discrepancy between the WHO and Turkish specific growth charts [27]. This problem may cause a major defect for the evaluation of malnutrition. Therefore, prospective trials are required to develop country specific growth charts and to detect malnutrition rates in different patient subgroups. This current study presented a relatively high rate of malnutrition for hospitalized pediatric urology patients. Screening questionnaires alone may be insufficient to investigate malnutrition for these patients. Therefore, anthropometric measurements should not be ignored in particularly certain patient subgroups such as VUR.

In conclusion, the current trial demonstrated that appropriate research should be conducted on malnutrition in hospitalized pediatric urology patients. Malnutrition was seen in approximately $30 \%$ of children admitted to our pediatric urology clinic. Particularly, children having VUR or UTI with neurogenic bladder had a higher malnutrition prevalence.

\section{REFERENCES}

[1] Ghosh-Jerath S, Singh A, Jerath N, Gupta S, Racine EF. Undernutrition and severe acute malnutrition in children. BMJ 2017;359: j4877. doi: 10.1136/bmj.j4877.

[2] Rytter MJ, Kolte L, Briend A, Friis H, Christensen VB. The immune system in children with malnutrition-a systematic review. PloSOne 2014;25;9:e105017. doi: 10.1371/journal. pone.0105017. eCollection 2014.

[3] Joosten KF, Hulst JM. Malnutrition in pediatric hospital patients: Current issues. Nutrition 2011;27:133-7. doi: 10.1016/j.nut.2010.06.001.

[4] Huysentruyt K, Alliet P, Muyshont L, et al. The STRONGkids nutritional screening tool in hospitalized children: A validation study. Nutrition 2013;29:1356-61. doi: 10.1016/j. nut.2013.05.008.

[5] Joosten KFM, Hulst JM. Prevalence of malnutrition in pediatric hospital patients. Curr Opin Pediatr 2008;2:590-6. doi: 10.1097/MOP.0b013e32830c6ede.

[6] Oztürk Y, Büyükgebiz B, Arslan N, Ellidokuz H. Effects of hospital stay on nutritional anthropometric data in Turkish children. J Trop Pediatr 2003; 49:189-90. doi: 10.1093/ tropej/49.3.189.

[7] Doğan Y, Erkan T, Yalvaç S, et al. Nutritional status of patients hospitalized in pediatric clinic. Turk J Gastroenterol 2005;16: 212-6.

[8] Durakbaşa ÇU, Fettahoğlu S, Bayar A, Mutus M, Okur H. The prevalence of malnutrition and effectiveness of STRONGkids tool in the identification of malnutrition risks among pediatric surgical patients. Balkan Med J 2014; 31:313-21. doi: 10.5152/ balkanmedj.2014.14374. Epub 2014.

[9] Agostoni C, Axelson I, Colomb V, et al. The need for nutrition support teams in pediatric units: a commentary by the ESPGHAN committee on nutrition. J Pediatr Gastroenterol Nutr 2005;41:8e11.

[10] Wonoputri N, Djais JT, Rosalina I. Validity of nutritional screening tools for hospitalized children J Nutr Metab. 2014;2014:143649. doi: 10.1155/2014/143649. Epub 2014 Sep 14.

[11] World Health Organization WHO. Child growth standards. Available from: http://www.who.int/childgrowth/software/en/

[12] World Health Organization WHO. Growth reference 5-19 years. Available from: http://www.who.int/growthref/tools/en

[13] Pollack MM, Ruttimann UE, Wiley JS. Nutritional depletions in critically ill children: associations with physiologic instability and increased quantity of care. J Parenter Enteral Nutr 1985; 9:309-13. doi:10.1177/014.860.7185009003309.

[14] Heyland DK, Montalvo M, MacDonald S, Keefe L, Su XY, Drover JW. Total parenteral nutrition in the surgical patient: a meta-analysis. Can J Surg 2001; 44:102-11.

[15] Barker LA, Gout BS, Crowe TC. Hospital malnutrition: prevalence, identification and impact on patients and the healthcare system. Int J Environ Res Public Health 2011; 8: 514-27. doi: 10.3390/ijerph8020514. Epub 2011 Feb 16.

[16] Middleton MH, Nazarenko G, Nivison-Smith I, Smerdely P. Prevalence of malnutrition and 12-month incidence of mortality in two Sydney teaching hospitals. Intern Med J 2001; 31: 455-61.

[17] Pawellek I, Dokoupil K, Koletzko B. Prevalence of malnutrition in paediatric hospital patients. ClinNutr 2008; 27: 72-6. doi: 10.1016/j.clnu.2007.11.001.

[18] Erkan T. Hastaneye yatan hastalarda beslenme riski değerlendirme yöntemleri. Türk Ped Arş 2014; 49: 276-81.

[19] Kondrup J, Allison SP, Elia M, Vellas B, Plauth M. ESPEN guidelines for nutrition screening 2002. Clin Nutr 2003;22:41521.

[20] Elia M, Zellipour L, Stratton RJ. To screen or not to screen for adult malnutrition. Clin Nutr 2005;24:867-4. doi: 10.1016/j. clnu.2005.03.004.

[21] Pablo AR, Izaga MA, Alday LA. Assessment of nutritional status on hospital admission: nutritional scores. Eur J Clin Nutr 2003;57:824-31. doi: 10.1038/sj.ejcn.1601616.

[22] Green SM, Watson R. Nutritional screening and assessment tools for use by nurses: literature review. J Adv Nurs 2005;50:69-83. doi: 10.1111/j.1365-2648.2004.03350.x.

[23] Moeeni V, Walls T, Day AS. The STRONGkids nutritional risk screening tool can be used by paediatric nurses to identify hospitalised children at risk. Acta Paediatr 2014; 103:e528-31. doi: 10.1111/apa.12768. Epub 2014 Sep 7.

[24] Joosten KF, Hulst JM. Nutritional screening tools for hospitalized children: methodological considerations. Clin Nutr 2014; 33:1-5. doi:10.106/j.clnu.2013.08.002. Epub 2013 Aug 31. 
[25] Hulst JM, Zwart H, Hop WC, Joosten KF. Duch national survey to test the STRONGkids nutritional risk screening tool in hospitalized children. ClinNutr 2010; 29: 106-11. doi: 10.1016/j.clnu.2009.07.006. Epub 2009 Aug 13.

[26] Beser OF, Cokugras FC, Erkan T, Kutlu T, Yagci RV. TUHAMAR Study Group. Evaluation of malnutrition development risk in hospitalized children. Nutrition 2018; 48: 40-7. doi: 10.1016/j. nut.2017.10.020. Epub 2017 Nov 29.

[27] Tutar E, Boran P, Öktem S, Tokuç G, Çalışkan B. Hastanede yatarak tedavi gören çocuklarda malnütrisyon: Ulusal Türk ve Dünya Sağlık Örgütü (DSÖ) çocuk büyüme standartlarının karşılaştırılması. Marmara Med J 2012;25:128-3. doi: 10.5472/ MMJ.2012.02242.1 